\title{
Membrane-associated GRP78 helps subgroup J avian leucosis virus enter cells
}

\author{
Lin Wang ${ }^{1,2+}$, Mei Mei ${ }^{1,2,5+}$, Aijian Qin ${ }^{1,2,3,4^{*}}$, Jianqiang Ye ${ }^{1,2,3}$, Kun Qian ${ }^{1,2,3}$ and Hongxia Shao ${ }^{1,2,3}$
}

\begin{abstract}
We previously identified chicken Annexin A2 (chANXA2) as a novel receptor for retrovirus avian leucosis virus subgroup J (ALV-J), using a DF1 cell line expressing the viral envelope (env) protein. To further probe whether other proteins participate in virus infection, we investigated several host proteins from co-immunoprecipitation with the DF1 cell line expressing viral env. Mass spectrometry analysis indicates that the chicken glucose-regulation protein 78 (chGRP78) of the DF1 membrane interacted with the ALV-J env protein. The results revealed that antibodies or siRNA to chGRP78 significantly inhibited ALV-J infection and replication, and over-expression of chGRP78 enabled the entry of ALV-J into non-susceptible cells. Taken together, these results are the first to report that chGRP78 functions to help ALV-J enter cells.
\end{abstract}

\section{Introduction}

Virus infection relies on the interaction between surface proteins and host receptors. In addition to virus receptors, other proteins may also interact with the virus and mediate infection. For example, HIV, a member of the retrovirus family, uses its glycoprotein gp120 to bind with CD4, CCR5 and CXCR4 as receptors to infect host cells [1-3]. Moreover, certain other proteins, such as CCR1, CCR8, CXCR6 (BONZO), GPR15 (BOB), GPR1, APJ, CX3CR1 (V28), CXCR5, and RDC1, also interact with gp120 and mediate virus entry into host cells [4-10]. Some of them were identified as co-receptors, which directly interact with virus and facilitate virus invasion to susceptible cells with greater efficiency.

ALV is another type of retrovirus. According to the antigenicity of its envelope glycoprotein, ALV can be divided into six subgroups (A, B, C, D, E, and J). The high variability of ALV-J's envelope (env) protein not only distinguishes it from other subgroups, but also results in alternations of the virus's pathogenicity, tumourigenicity and host range [11]. The env protein's surface unit (SU) is

\footnotetext{
*Correspondence: aijian@yzu.edu.cn

${ }^{\dagger}$ Lin Wang and Mei Mei contributed equally to this work

${ }^{2}$ Key Laboratory of Jiangsu Preventive Veterinary Medicine, Yangzhou

University, No. 12 East Wenhui Road, Yangzhou 225009, China

Full list of author information is available at the end of the article
}

a virus receptor binding-determining region. Currently, four cellular receptors for ALV have been identified. Tva [12], tvc [13], and chNHE1 [14] interact with the env proteins of ALV-A, C, and J, respectively. Recent research has identified chANXA2 as another receptor that is specific to ALV-J [15]. To further probe whether there are other proteins that specifically interact with ALV-J's env protein to mediate viral infection, we used DF1 cell lines that over express ALV-J's env protein to capture proteins of interest. Using immunological and molecular biological approaches, we identified $78-\mathrm{kDa}$ glucose-regulated protein (GRP78) as a novel host protein that interacts with the env of ALV-J and is involved in the infection of DF1 cells by ALV-J. GRP78 is also referred to as Bip/ HSPA5. This protein consists of membrane-associated $[16,17]$ and trans-membrane segments [18]. More than a ER stress-regulating chaperone [19], GRP78 participates in several biological or immunological processes and extensively influences virus infection or pathogenicity [20-23]. Meanwhile, GRP78 has been identified as a receptor for different types of virus, such as coxsackie $B$ [24] and dengue fever virus [25], which directly interact with viral protein. Our results firmly establish that GRP78 can interact with the env of ALV-J to aid the entry of ALV-J into cells. 


\section{Materials and methods Cells and virus}

DF1 cells and pcDNA-env_DF1 cells [26] were maintained in Dulbecco's modified Eagle's medium (DMEM) supplemented with $5 \%$ fetal bovine serum and $1 \%$ antibiotics. HEK293T cells and GEF (Goose embryo fibroblast) cells were maintained in DMEM supplemented with $10 \%$ fetal bovine serum and 1\% antibiotics. The ALV-J strain (JS09GY07) and ALV-A strain (AH10) were preserved in our laboratory.

\section{Antibodies and primers}

Monoclonal antibody JE9, which specifically recognizes ALV-J's gp85, was employed in the indirect immunofluorescence (IFA), Western blot and immunoprecipitation experiments to detect or capture ALV-J's env protein [27]. Monoclonal antibody 5D3, which specifically recognizes ALV's group specific protein p27, was employed in Western blot. A polyclonal antibody for chGRP78 was purchased from Santa Cruz Biotechnology, the product code is sc-1051 (Dallas, USA). Monoclonal antibody chicken $\beta$-actin (Santa Cruz Biotechnology, Dallas, USA) was used as an internal control in the Western blot. FITC-labelled goat anti-mouse antibody (Sigma, St. Louis, MO, USA) and HRP-labelled goat anti-mouse antibody (Sigma) were used as secondary antibodies. The primers used in the real-time PCR and the siRNA used to inhibit the expression of chGRP78 are listed in Table 1. The siRNA was synthesized by Invitrogen (Carlsbad, California, USA).

\section{Co-immunoprecipitation and mass spectrometry}

pcDNA-env_DF1 cells in $100 \mathrm{~mm}$ dishes were harvested by scraping with a rubber policeman and homogenised

Table 1 Sequences of the primers used for real-time PCR and the siRNA against chGRP78

\begin{tabular}{|c|c|c|}
\hline & Name & Sequence $\left(5^{\prime}-3^{\prime}\right)$ \\
\hline \multirow[t]{8}{*}{ RT-PCR } & ALV-J gp37 & Forward:TGCGTGCGTGGTATTATTTC \\
\hline & & Reverse: AATGGTGAGGTCGCTGACTGT \\
\hline & chGRP78 & Forward: GACGATGAGGAGAAAAAGGAG \\
\hline & & Reverse: TGAATACACCCACACAAGAAT \\
\hline & Chicken 18s & Forward:TCAGATACCGTCGTAGTTCC \\
\hline & & Reverse: TTCCGTCAATTCCTTTAAGTT \\
\hline & Human $\beta$-actin & Forward: CACGAAACTACCTTCAACTCC \\
\hline & & Reverse: CATACTCCTGCTTGCTGATC \\
\hline \multirow[t]{6}{*}{ siRNA } & 366 & Forward: AGGACAUCAAGUAUCUGCCCUUCAA \\
\hline & & Reverse: UUGAAGGGCAGAUACUUGAUGUCCU \\
\hline & 611 & Forward: GGGUUGAACGUGAUGCGCAUUAUUA \\
\hline & & Reverse: UAAUAAUGCGCAUCACGUUCAACCC \\
\hline & 519 & Forward: CCCACAGAUUGAAGUUACCUUUGAA \\
\hline & & Reverse: UUCAAAGGUAACUUCAAUCUGUGGG \\
\hline
\end{tabular}

with NP-40 lysis buffer containing $25 \mathrm{mM}$ Tris, $150 \mathrm{mM}$ $\mathrm{NaCl}, 1 \mathrm{mM}$ EDTA, 1\% NP-40, 5\% glycerol (pH7.4) and a protease inhibitor cocktail (Roche, Basel, Switzerland) incubating for $20 \mathrm{~min}$, then the membrane proteins in the supernatant were sedimented by an additional spin at $13200 \mathrm{~g}$ for $1 \mathrm{~h}$ at $4{ }^{\circ} \mathrm{C}$ and resuspended in $1 \% \mathrm{NP}-40$ lysis buffer. The membrane proteins from the pcDNAenv_DF1 cells were immunoprecipitated with the monoclonal antibody JE9, which is specific to ALV-J Env and Resin A (Thermo Scientific, Massachusetts, USA). Precipitated proteins were separated by SDS-PAGE. The gel was stained with a Silver Stain Kit for MS (Thermo Scientific). The bands of interests were collected and analysed using mass spectrometry [15].

\section{Antibody blocking assay}

The DF1 cells were pre-treated with anti-GRP78 antibody which was diluted with DMEM at a concentration of 5 , 25 and $50 \mu \mathrm{g} / \mathrm{mL}$ at $37{ }^{\circ} \mathrm{C}$ for $2 \mathrm{~h}$ and subsequently challenged with ALV-J at a MOI of 5 and incubated at $37{ }^{\circ} \mathrm{C}$ for $2 \mathrm{~h}$. The DF1 cells were pre-treated with anti-GRP78 antibody which was diluted with DMEM at a concentration of $50 \mu \mathrm{g} / \mathrm{mL}$ and subsequently challenged with ALV-A at a MOI of 5 and incubated at $37^{\circ} \mathrm{C}$ for $2 \mathrm{~h}$. The cells were treated with acid glycine ( $\mathrm{pH} 3.0$ ) for $1 \mathrm{~min}$ to inactivate the non-internalized virus. After a wash with PBS, the cells were maintained in DMEM with $1 \%$ foetal calf serum and antibody at the concentrations mentioned above for $48 \mathrm{~h}$. The replication of ALV-J in the treated cells was analysed with IFA, real-time PCR, Tissue culture infective dose $50\left(\mathrm{TCID}_{50}\right)$ and Western blot, and the replication of ALV-A in the treated cells was analysed with Western blot.

\section{RNA interference assay}

DF1 cells were transfected with stealth siRNA (50 pmol) against chGRP78. Six hours later, the cells were infected with ALV-J at a MOI of 1 and incubated at $37{ }^{\circ} \mathrm{C}$ for $2 \mathrm{~h}$. Then, the cells were maintained in DMEM with $1 \%$ fetal bovine serum for $72 \mathrm{~h}$. The interference effects of the siRNA directed against chGRP78 were evaluated with real-time PCR. Simultaneously, the replication of ALV-J in the DF1 cells was analysed with Western blot and $\mathrm{TCID}_{50}$ titration of the ALV-J in the collected cell culture supernatant.

\section{Infection of chGRP78-transfected cells with different concentration}

First, we inserted the 1982 bp full-length chGRP78 coding sequence into pcDNA3.1; human 293T cells were transfected with different concentrations of pcDNA3.1-chGRP78 $(0.5,1.5,3.0$ and $4.5 \mu \mathrm{g}$ respectively) and also transfected with $4.5 \mu \mathrm{g}$ pcDNA3. 1 as the control. Forty-eight hours later, 
the transfected cells were infected with ALV-J at a MOI of ten for $2.5 \mathrm{~h}$. Subsequently the cells were treated with acid glycine (pH 3.0) for $1 \mathrm{~min}$ and washed three times with PBS. Then, the 293T cells were maintained in DMEM with $1 \%$ foetal bovine serum for $48 \mathrm{~h}$. The cells were extracted RNA and elevated with real-time PCR.

\section{Infection of chGRP78-transfected cells}

GEF cells were transfected with pcDNA3.1-chGRP78. The cells were transfected with pcDNA3.1 as the control. Forty-eight hours later, the transfected cells were infected with ALV-J at a MOI of ten for $2.5 \mathrm{~h}$. Then, the GEF cells were maintained in DMEM with $1 \%$ foetal bovine serum. After 6 days later, the replication of ALV-J was analysed with IFA using JE9 antibody.

\section{Real-time PCR}

RNA was isolated from whole-cell lysates using a Total RNA Miniprep Kit (Axygen, California, USA). The RNA was reverse transcribed using a PrimerScript RT Reagent Kit (Takara, Kusatsu, Japan) with primers targeting the ALV-J gp37 and chGRP78 coding sequences. Chicken $18 \mathrm{~s}$ and human $\beta$-actin were used as an internal control in the real-time PCR. Fold change of target gene RNA level was calculated with the $2^{-\triangle \triangle \mathrm{Ct}}$ method. GraphPad Prism 5.0 (GraphPad Software) was used to analyse the data. Data is expressed in column style with mean $\pm \mathrm{SD}$ and statistic variance was analysed using the One-way ANOVA test. $P<0.05$ was considered as significant.

\section{$\mathrm{TCID}_{50}$ assay}

To determine the $\mathrm{TCID}_{50}$ level of the ALV-J in the collected supernatants, DF1 cells were plated in 96-well plates $\left(2.5 \times 10^{4}\right.$ cells/well $)$. Twenty-four hours later, the supernatants from antibody blocking assays or RNA interference assays were continuously diluted from 10- to $10^{10}$-fold. Each dilution was used to infect the DF1 cells in eight duplicate wells. After incubation at $37{ }^{\circ} \mathrm{C}$ for $2.5 \mathrm{~h}$, the supernatants were replaced with DMEM containing $1 \%$ foetal bovine serum. Seven days later, the cells were immobilized, and IFA was conducted with JE9. The final dilution of virus TCID $_{50}$ was calculated according to the Reed-Muench formula.

\section{IFA}

The treated DF1 cells and GEF cells were immobilized with $4 \%$ paraformaldehyde and then treated with $0.25 \%$ Triton X-100. After incubation with PBS containing $2 \%$ BSA for $45 \mathrm{~min}$, the cells were incubated with JE9 as the primary antibody. Then, FITC-labelled goat anti-mouse antibody was used as the secondary antibody. Finally, Hoechst 33342 was used to stain the cell nuclei at room temperature for $2 \mathrm{~min}$. The cells were stored in 50\% glycerine, and the fluorescence signals were observed under confocal microscopy.

\section{Western blot of env expression in DF1 cells}

The expression levels of ALV-J env protein were also detected with Western blot. Following infection with ALV-J for $48 \mathrm{~h}$, the DF1 cells were harvested and lysed with $1 \% \mathrm{NP}-40$ and a protease inhibitor cocktail. The cell lysates were used for SDS-PAGE and then transferred to nitrocellulose membranes for Western blot analysis. JE9, 5D3 and $\beta$-actin were employed as the first antibody respectively, and HRP-labelled goat anti-mouse antibody was employed as the second antibody. The membrane was finally immersed in Super Signal West Pico (Thermo, Massachusetts, USA), and the chemiluminescent signals were observed with a FluorChemE imaging system (Protein Simple, California, USA).

\section{Results}

chGRP78 bound the env protein of ALV-J

To identify a novel ALV-J-binding protein in the DF1 cells, the pcDNA-env_DF1 cell line expressing ALV-J env protein was used in co-immunoprecipitation assays (co-IP). The pcDNA-env_DF1 cells were lysed and then performed immunoprecipitation with the single monoclonal antibody (mAb) JE9, which is specific to ALV-J env. Silver staining for SDS-PAGE of the immunoprecipitation revealed several different bands in the lysate that was immunoprecipitated with ALV-J-specific mAb JE-9 and not with the control antibody (Figure 1). Mass spectrometry further revealed that one of these bands was chGRP78 which was 78-kDa.

The antibody against chGRP78 significantly inhibited ALV-J infection and replication

We used antibodies against chGRP78 to perform blocking assays to evaluate the effects of chGRP78 on ALV-J

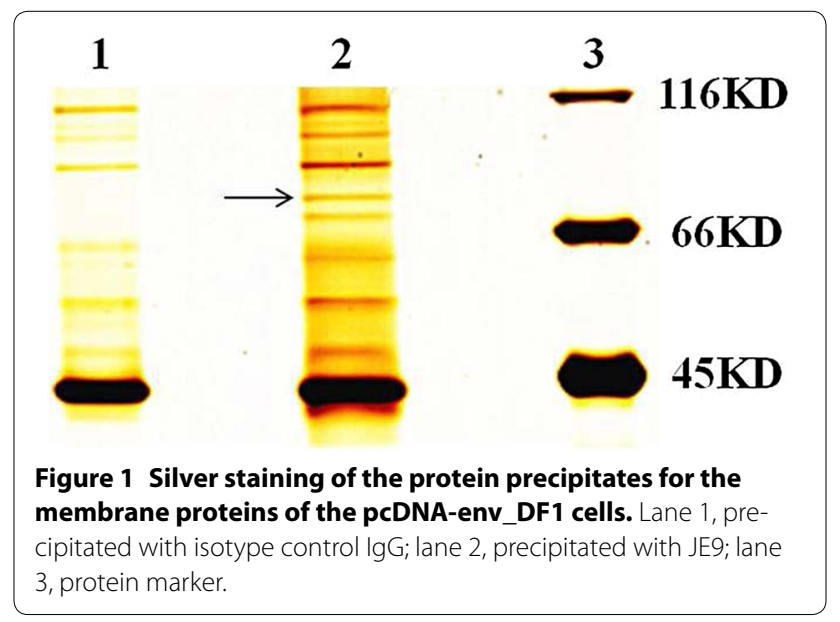


infection in DF1 cells. Our results revealed that the viral infection/replication of ALV-J was significantly inhibited in groups that had been treated with anti-GRP78. As shown in Figure 2A, there was little visible immunofluorescence in the cells that were treated with 50 or $25 \mu \mathrm{g} / \mathrm{mL}$ of the antibody against chGRP78 in the IFA. Moreover, only a few positive cells were found among the cells that were treated with $5 \mathrm{mg} / \mathrm{mL}$ of antibody against chGRP78. In contrast, many positive cells were found among the cells that were treated with the control IgG and among the untreated cells., the viral titres of the cells that were treated with 50 and $25 \mu \mathrm{g} / \mathrm{mL}$ of antibody against chGRP78 were approximately 50 - and 10-fold less, respectively, than those of the cells that were treated with the control IgG being consistent with the IFA results (Figure 2B). The inhibitory effect on ALV-J infection/replication conferred by the antibody against chGRP78 was also confirmed by Western blot (Figure 2C). As a viral control, we also performed a blocking assay for ALV-A infection in the DF1 cells. As described in Figure 2D, the p27 expression levels of ALV-A in the groups that were treated with the antibody against chGRP78 were similar to those of the mock group, which indicates that the antibody against chGRP78 could not inhibit ALV-A infection/replication in DF1 cells. These data clearly demonstrate that blocking chGRP78 with a specific antibody can effectively and specifically inhibit the infection/ replication of ALV-J.

\section{RNA interference with chGRP78 in DF1 cells inhibited ALV-J infection}

To further demonstrate that the chGRP78 protein plays an important role in ALV-J infection, an RNA interference assay was performed. Three pairs of siRNA were employed to reduce chGRP78 expression. Real-time PCR indicated that the mRNA level of chGRP78 was significantly inhibited in the DF1 cells (Figure 3A). Moreover, the Western blot results for the ALV-J envelope protein

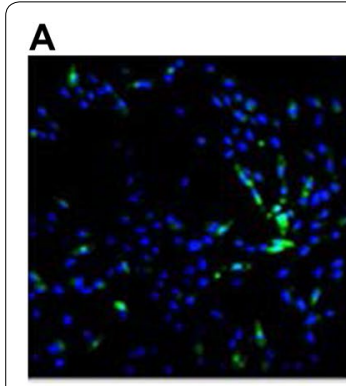

ALV-J

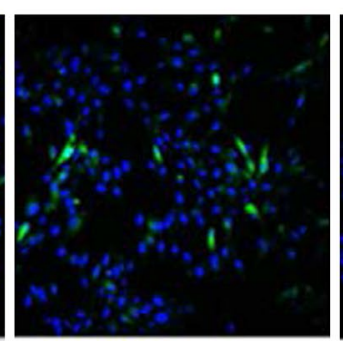

ALV-J +goat IgG $(50 \mu \mathrm{g} / \mathrm{mL})$
B

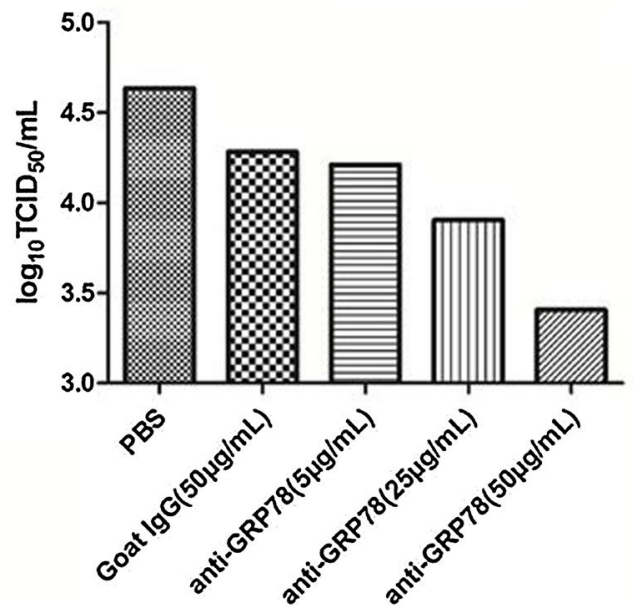

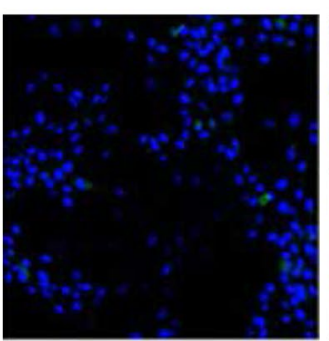

ALV-J

tanti-GRP78

$(5 \mu \mathrm{g} / \mathrm{mL})$

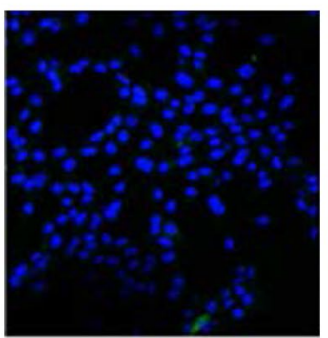

ALV-J

tanti-GRP78

$(25 \mu \mathrm{g} / \mathrm{mL})$

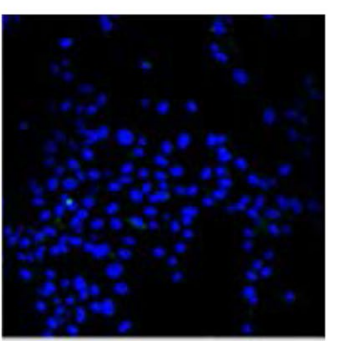

ALV-J

tanti-GRP78

$(50 \mu \mathrm{g} / \mathrm{mL})$

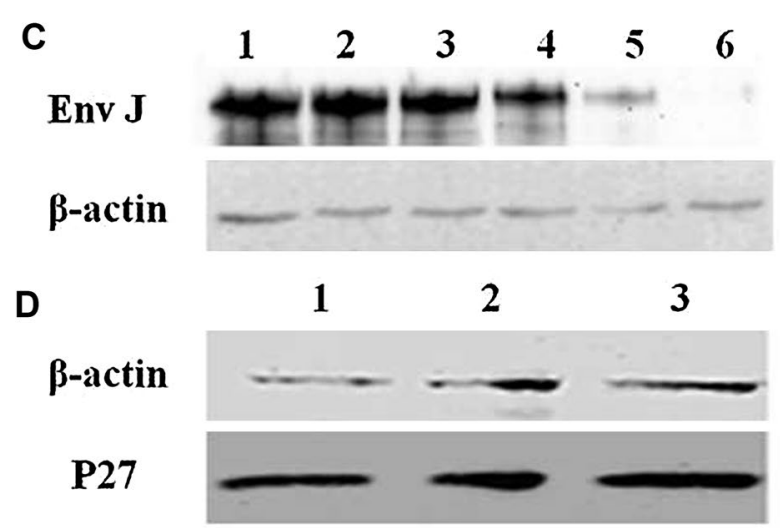

Figure 2 Inhibition of ALV-J infection by antibodies to GRP78. The DF1 cells that had been pre-treated with antibodies against chGRP78 were infected with ALV-J, and ALV-J replication in the treated cells was analysed. A IFA analysis using JE9; B TCID ${ }_{50}$ analysis for viral titres; C Western blot analysis for the expression of env from ALV-J in the DF1 cells that had been treated with antibodies. Lane 1, blank; lane 2, mock; lane 3, goat lgG $(50 \mu \mathrm{g} / \mathrm{mL})$; lane 4, anti-GRP78 (5 $\mathrm{g} / \mathrm{mL})$; lane 5, anti-GRP78 (25 $\mu \mathrm{g} / \mathrm{mL})$; lane 6, anti-GRP78 $(50 \mu \mathrm{g} / \mathrm{mL})$; D Western blot analysis of the expression of ALV-A p27 in the DF1 cells treated with antibodies. Lane 1, anti-GRP78 $(50 \mu \mathrm{g} / \mathrm{mL})$; lane 2, goat lgG $(50 \mu \mathrm{g} / \mathrm{mL})$; lane 3, mock. 

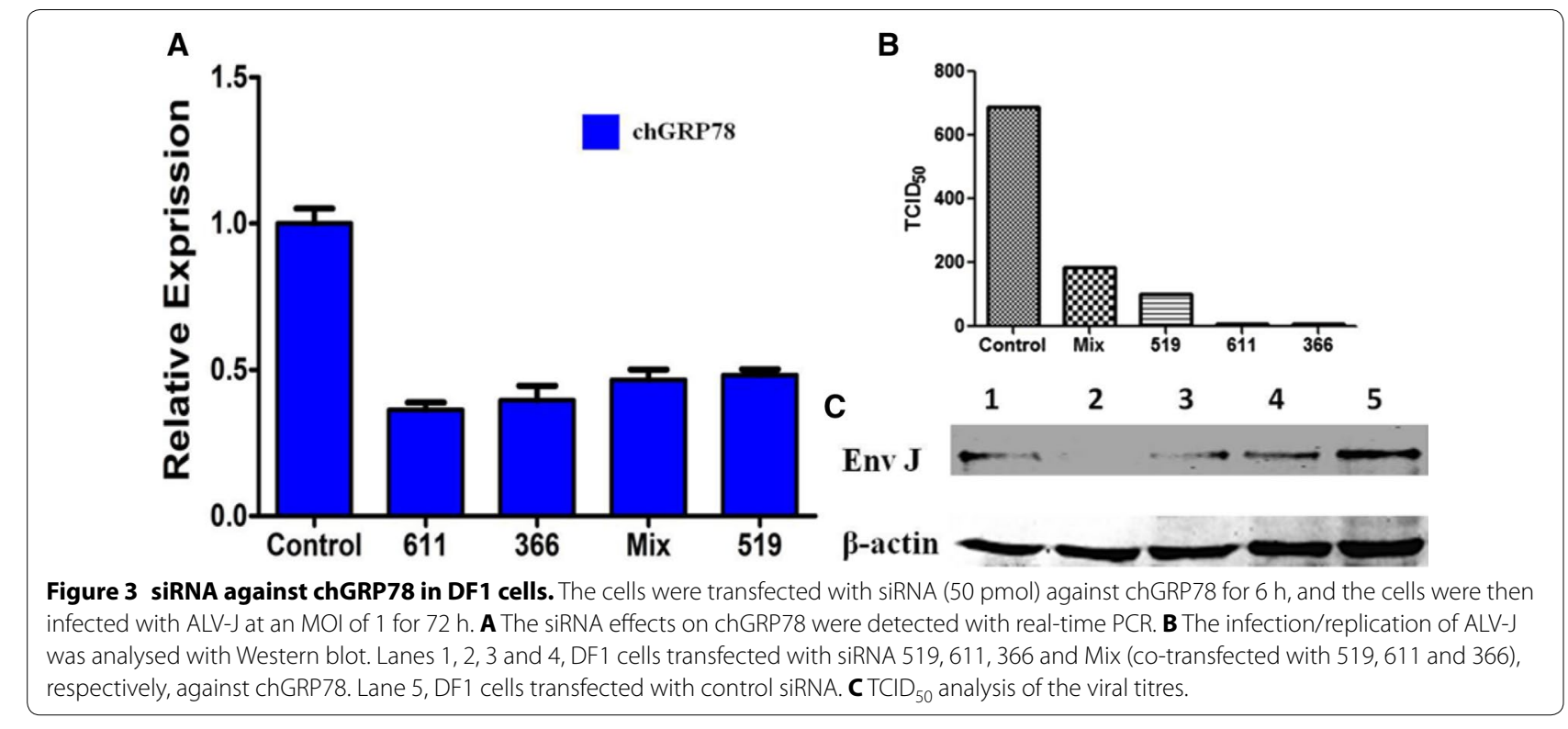

and the $\mathrm{TCID}_{50}$ titration of ALV-J in the DF1 cell supernatant revealed that virus replication was inhibited (Figures $3 \mathrm{~B}$ and $\mathrm{C}$ ). These results suggest that chGRP78 plays a significant role in the process of ALV-J infection of the host cell.

\section{chGRP78 over-expression allowed the ALV-J into non-permissible cells}

Human 293T cells, which are non-susceptible to ALV$J$, were transfected with different concentrations of pcDNA3.1-chGRP78 $(0.5,1.5,3.0$ and $4.5 \mu \mathrm{g})$ respectively, and transfected with $4.5 \mu \mathrm{g}$ pcDNA3.1 as the control. After $48 \mathrm{~h}$, the transfected cells were infected with ALV-J at an MOI of 5 . The viral gp37 gene was detected by realtime PCR. We found that the mRNA level of ALV-J gp37 increased apparently with the concentration increasing, a significant positive correlation (Figure 4A). To further confirm this finding, we also transfected chGRP78 into GEF cells in which the ALV-J could not grow [28]. After $48 \mathrm{~h}$, the transfected GEF were infected with ALV-J. The GEF cells were maintained in DMEM medium containing $1 \%$ foetal bovine serum for 6 days. Interestingly, as the IFA shows in Figure 4B, virus was recovered from the GEF cells transfected with chGRP78, but no virus was obtained from the GEF cells that were transfected with the control plasmid. Then, the infected GEF and supernatant were collected to recover ALV-J on DF1 cells. As excepted, the virus could be detected on DF1 cells by IFA.

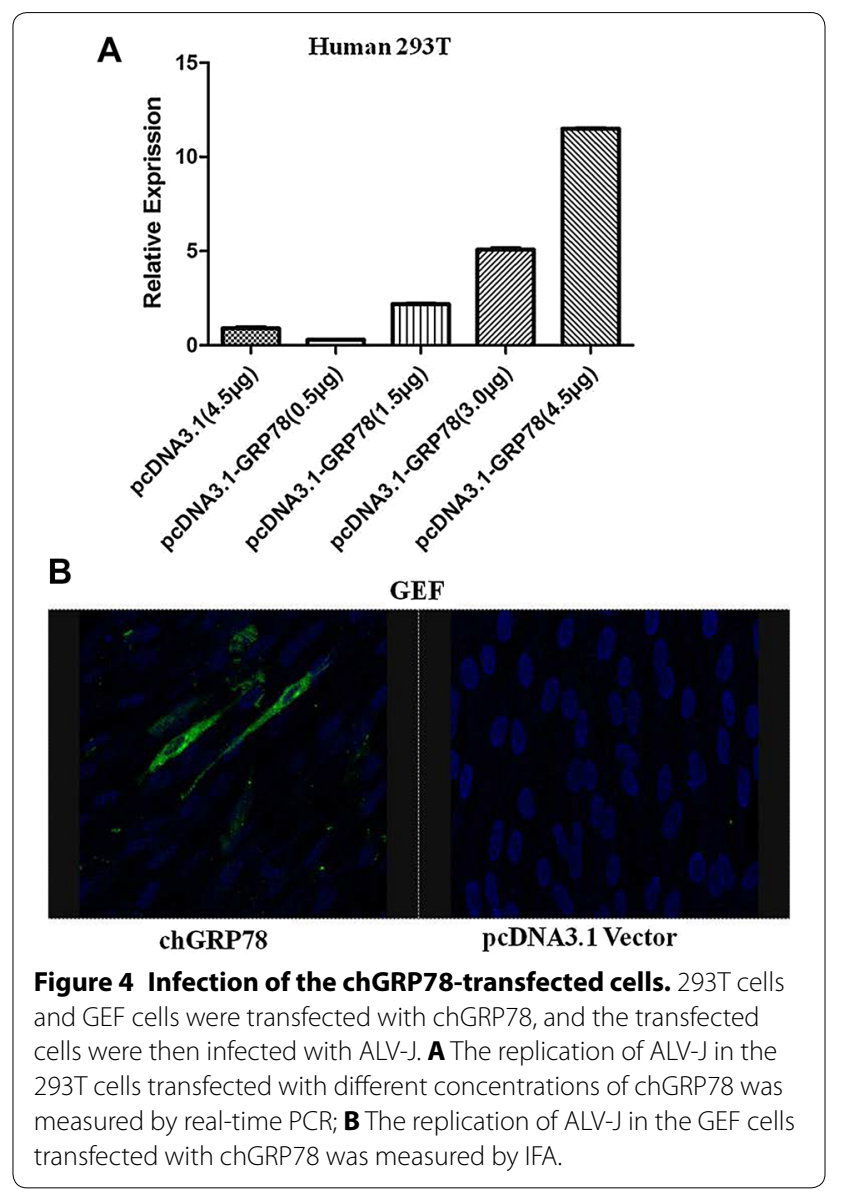


Together, these data demonstrate that the over-expression of chGRP78 in 293T or GEF cells can support ALV-J entry into these ALV-J non-permissible cells.

\section{Discussion}

Virus attachment and entrance into host cells rely on host receptors. Occasionally, other host proteins may assist in the virus infection. For example, host glycosaminoglycans have been implicated in the mediation of the attachment and entry of mosquito-borne flaviviruses into mammalian cells $[29,30]$; however, the corresponding function has not been elucidated. Previous studies with Japanese encephalitis virus (JEV) have reported the possible involvements of a $57-\mathrm{kDa}$ protein derived from BHK-21 cells, the GAG protein from $\mathrm{CHO}$ cells and a 74-kDa molecule from Vero cells as possible receptors [31]. Subsequently, another report hypothesized that vimentin, an important cellular protein, is responsible for the attachment and entry of JEV into porcine cells [32].

Similarly, in addition to the identified host receptors chANXA2 and chNHE1, we found that chGRP78 is another important protein for AVL-J infection of DF1 cells. chGRP78 is a member of the HSP70 family that is located in both the membrane and plasma of various types of cells [33]. Despite its immune and biological functions [34], substantial research has found that chGRP78 is a receptor for pathogens such as dengue virus [35], coxsackie virus [24] and herpes simplex virus [36]. The roles of chGRP78 in the infections of other pathogens have been elucidated [37-39]. Collectively, this research indicates that chGRP78 may also play a role in avian pathogen infection. In the present study, we found that ALV-J replication was significantly inhibited when the DF1 cells were blocked with polyclonal antibody against chGRP78 or the expression of chGRP78 was interfered with via siRNA. The inhibition efficiency in DF1 cells is the same as that in chANXA2 [15]. This finding indicates that chGRP78 may have a similar function as chANXA2 in AVL-J infection. The over-expression of chGRP78 could permit the entry of ALV-J into 293T cells. Our study shows that the RNA amount of gp37 was increased in the first $48 \mathrm{~h}$ in $293 \mathrm{~T}$ cells which were transfected with chGRP78, while other conditions were the same. In addition, ALV-J infection efficiency was also dose-dependent on chGRP78 expression in 293T cells. The gain of ALV-J susceptibility in GEF further supports the conclusion that chGRP78 may be a novel functional co-receptor for ALV-J infection. This finding indicates the importance of chGRP78 in AVL-J entry into host cells. However, the potential interaction among chNHE1, chANXA2 and chGRP78 need to be investigated, and the mechanism by which chGRP78 introduced ALV-J into the host cells remains unclear. Both of these issues are the subject of our future research. This research will also provide additional information regarding retrovirus prevention and control.

\section{Competing interests}

The authors declare that they have no competing interests.

\section{Authors' contributions}

$M M, A Q, K Q$ and JY conceived and designed the experiments; LW and MM performed the experiments. LW, MM, AQ, KQ, HS and JY analysed the data. LW, MM, HXS and JY contributed reagents/materials/analysis tools. LW and AQ contributed to the writing of the manuscript. LW prepared the figures. All authors read and approved the final manuscript.

\section{Acknowledgements}

This study was supported by the National Natural Science Foundation of China (31201881, 31472171), National Science and Technology Transformation Fund Project (2014GB2C100292), National key research and development plan (2016YFD0500803), The Priority Academic Program Development of Jiangsu Higher Education, Institutions of the Chinese Special Fund for Agroscientific Research in the Public Interest (201203055) and Special Foundation for State Basic Research Program of China (2013FY113300-4).

\section{Author details}

${ }^{1}$ Ministry of Education Key Lab for Avian Preventive Medicine, Yangzhou University, No. 12 East Wenhui Road, Yangzhou 225009, Jiangsu, China. ${ }^{2}$ Key Laboratory of Jiangsu Preventive Veterinary Medicine, Yangzhou University, No. 12 East Wenhui Road, Yangzhou 225009, China. ${ }^{3}$ Jiangsu Co-innovation Center for Prevention and Control of Important Animal Infectious Diseases and Zoonoses, Yangzhou University, No. 12 East Wenhui Road, Yangzhou 225009, China. ${ }^{4}$ Jiangsu Key Lab of Zoonosis, Yangzhou University, No. 12 East Wenhui Road, Yangzhou 225009, China. ${ }^{5}$ Present Address: National Research Center of Engineering and Technology for Veterinary Biologicals, Jiangsu Academy of Agricultural Sciences, Nanjing 210014, Jiangsu, China.

Received: 17 March 2016 Accepted: 7 July 2016

Published online: 06 September 2016

\section{References}

1. Zhou J, Satheesan S, Li H, Weinberg MS, Morris KV, Burnett JC, Rossi JJ (2015) Cell-specific RNA aptamer against human CCR5 specifically targets HIV-1 susceptible cells and inhibits HIV-1 infectivity. Chem Biol 22:379-390

2. Veillette M, Coutu M, Richard J, Batraville LA, Dagher O, Bernard N, Tremblay C, Kaufmann DE, Roger M, Finzi A (2015) The HIV-1 gp120 CD4bound conformation is preferentially targeted by antibody-dependent cellular cytotoxicity-mediating antibodies in sera from HIV-1-infected individuals. J Virol 89:545-551

3. Das D, Maeda K, Hayashi Y, Gavande N, Desai DV, Chang SB, Ghosh AK, Mitsuya $H$ (2015) Insights into the mechanism of inhibition of CXCR4: identification of piperidinylethanamine analogs as Anti-HIV-1 inhibitors. Antimicrob Agents Chemother 59:1895-1904

4. Calado M, Matoso P, Santos-Costa Q, Espirito-Santo M, Machado J, Rosado L, Antunes F, Mansinho K, Lopes MM, Maltez F, Santos-Ferreira MO, Azevedo-Pereira JM (2010) Coreceptor usage by HIV-1 and HIV-2 primary isolates: the relevance of CCR8 chemokine receptor as an alternative coreceptor. Virology 408:174-182

5. Cagigi A, Mowafi F, Phuong Dang LV, Tenner-Racz K, Atlas A, Grutzmeier S, Racz P, Chiodi F, Nilsson A (2008) Altered expression of the receptorligand pair CXCR5/CXCL13 in B cells during chronic HIV-1 infection. Blood 112:4401-4410

6. Passam AM, Sourvinos G, Krambovitis E, Miyakis S, Stavrianeas N, Zagoreos I, Spandidos DA (2007) Polymorphisms of Cx(3)CR1 and CXCR6 receptors in relation to HAART therapy of HIV type 1 patients. AIDS Res Hum Retrovir 23:1026-1032 
7. Garin A, Tarantino N, Faure S, Daoudi M, Lecureuil C, Bourdais A, Debre P, Deterre P, Combadiere C (2003) Two novel fully functional isoforms of CX3CR1 are potent HIV coreceptors. J Immunol 171:5305-5312

8. Fan X, Zhou N, Zhang X, Mukhtar M, Lu Z, Fang J, DuBois GC, Pomerantz RJ (2003) Structural and functional study of the apelin-13 peptide, an endogenous ligand of the HIV-1 coreceptor, APJ. Biochemistry 42:10163-10168

9. Wade-Evans AM, Russell J, Jenkins A, Javan C (2001) Cloning and sequencing of cynomolgus macaque CCR3, GPR15, and STRL33: potential coreceptors for HIV type 1, HIV type 2, and SIV. AIDS Res Hum Retrovir 17:371-375

10. Shimizu N, Soda Y, Kanbe K, Liu HY, Mukai R, Kitamura T, Hoshino H (2000) A putative $\mathrm{G}$ protein-coupled receptor, $\mathrm{RDC1}$, is a novel coreceptor for human and simian immunodeficiency viruses. J Virol 74:619-626

11. Cicala C, Arthos J (2014) Virion attachment and entry: HIV gp120 Env biotinylation, gp120 Env, or integrin ligand-binding assay. Methods Mol Biol 1087:3-12

12. Bates P, Young JA, Varmus HE (1993) A receptor for subgroup A Rous sarcoma virus is related to the low density lipoprotein receptor. Cell 74:1043-1051

13. Elleder D, Stepanets V, Melder DC, Senigl F, Geryk J, Pajer P, Plachy J, Hejnar J, Svoboda J, Federspiel MJ (2005) The receptor for the subgroup C avian sarcoma and leukosis viruses, Tvc, is related to mammalian butyrophilins, members of the immunoglobulin superfamily. J Virol 79:10408-10419

14. Chai $\mathrm{N}$, Bates $\mathrm{P}$ (2006) $\mathrm{Na}+/ \mathrm{H}+$ exchanger type 1 is a receptor for pathogenic subgroup J avian leukosis virus. Proc Natl Acad Sci USA 103:5531-5536

15. Mei M, Ye J, Qin A, Wang L, Hu X, Qian K, Shao H (2015) Identification of novel viral receptors with cell line expressing viral receptor-binding protein. Sci Rep 5:7935

16. Gonzalez-Gronow M, Selim MA, Papalas J, Pizzo SV (2009) GRP78: a multifunctional receptor on the cell surface. Antioxid Redox Signal 11:2299-2306

17. Quinones QJ, de Ridder GG, Pizzo SV (2008) GRP78: a chaperone with diverse roles beyond the endoplasmic reticulum. Histol Histopathol 23:1409-1416

18. Reddy RK, Mao C, Baumeister P, Austin RC, Kaufman RJ, Lee AS (2003) Endoplasmic reticulum chaperone protein GRP78 protects cells from apoptosis induced by topoisomerase inhibitors: role of ATP binding site in suppression of caspase-7 activation. J Biol Chem 278:20915-20924

19. Lee AS (2005) The ER chaperone and signaling regulator GRP78/BiP as a monitor of endoplasmic reticulum stress. Methods 35:373-381

20. Lyoo HR, Park SY, Kim JY, Jeong YS (2015) Constant up-regulation of BiP/ GRP78 expression prevents virus-induced apoptosis in BHK-21 cells with Japanese encephalitis virus persistent infection. Virol J 12:32

21. Wei D, Li NL, Zeng Y, Liu B, Kumthip K, Wang TT, Huo D, Ingels JF, Lu L, Shang J, Li K (2016) The molecular chaperone GRP78 contributes to tolllike receptor 3 -mediated innate immune response to hepatitis $C$ virus in hepatocytes. J Biol Chem 291:12294-12309

22. Zhang $X$, Zhang $R$, Yang $H$, Xiang $Q$, Jiang $Q$, He Q, Zhang $T$, Chen C, Zhu H, Wang Q, Ning Q, Li Y, Lei P, Shen G (2016) Hepatitis B virus enhances cisplatin-induced hepatotoxicity via a mechanism involving suppression of glucose-regulated protein of $78 \mathrm{kDa}$. Chem Biol Interact 254:45-53

23. Zhou Y, Qi B, Gu Y, Xu F, Du H, Li X, Fang W (2016) Porcine circovirus 2 deploys PERK pathway and GRP78 for its enhanced replication in PK-15 cells. Viruses 8:E56
24. Triantafilou K, Fradelizi D, Wilson K, Triantafilou M (2002) GRP78, a coreceptor for coxsackievirus A9, interacts with major histocompatibility complex class I molecules which mediate virus internalization. J Virol 76:633-643

25. Jindadamrongwech S, Thepparit C, Smith DR (2004) Identification of GRP 78 (BiP) as a liver cell expressed receptor element for dengue virus serotype 2. Arch Virol 149:915-927

26. Ye J, Qin A, Shao H, Liu H, Jin W, Liu Y (2005) Development of chicken embryo fibroblast cell line resistant to J subgroup avian leukosis virus (ALV-J) infection. Bing Du Xue Bao 21:456-460

27. Qin A, Lee LF, Fadly A, Hunt H, Cui Z (2001) Development and characterization of monoclonal antibodies to subgroup J avian leukosis virus. Avian Dis 45:938-945

28. Reinisova M, Plachy J, Kucerova D, Senigl F, Vinkler M, Hejnar J (2016) Genetic diversity of NHE1, receptor for subgroup J avian leukosis virus, in domestic chicken and wild Anseriform species. PLoS One 11:e0150589

29. Chen Y, Maguire T, Hileman RE, Fromm JR, Esko JD, Linhardt RJ, Marks RM (1997) Dengue virus infectivity depends on envelope protein binding to target cell heparan sulfate. Nat Med 3:866-871

30. Lee E, Lobigs M (2000) Substitutions at the putative receptor-binding site of an encephalitic flavivirus alter virulence and host cell tropism and reveal a role for glycosaminoglycans in entry. J Virol 74:8867-8875

31. Das S, Laxminarayana SV, Chandra N, Ravi V, Desai A (2009) Heat shock protein 70 on Neuro2a cells is a putative receptor for Japanese encephalitis virus. Virology 385:47-57

32. Das S, Ravi V, Desai A (2011) Japanese encephalitis virus interacts with vimentin to facilitate its entry into porcine kidney cell line. Virus Res 160:404-408

33. Buchkovich NJ, Maguire TG, Paton AW, Paton JC, Alwine JC (2009) The endoplasmic reticulum chaperone BiP/GRP78 is important in the structure and function of the human cytomegalovirus assembly compartment. J Virol 83:11421-11428

34. Galligan JJ, Fritz KS, Backos DS, Shearn CT, Smathers RL, Jiang H, MacLean KN, Reigan PR, Petersen DR (2014) Oxidative stress-mediated aldehyde adduction of GRP78 in a mouse model of alcoholic liver disease: functional independence of ATPase activity and chaperone function. Free Radic Biol Med 73:411-420

35. Wati S, Soo ML, Zilm P, Li P, Paton AW, Burrell CJ, Beard M, Carr JM (2009) Dengue virus infection induces upregulation of GRP78, which acts to chaperone viral antigen production. J Virol 83:12871-12880

36. Wu YP, Chang CM, Hung CY, Tsai MC, Schuyler SC, Wang RY (2011) Japanese encephalitis virus co-opts the ER-stress response protein GRP78 for viral infectivity. Virol I 8:128

37. Liu R, Li X, Gao W, Zhou Y, Wey S, Mitra SK, Krasnoperov V, Dong D, Liu S, Li D, Zhu G, Louie S, Conti PS, Li Z, Lee AS, Gill PS (2013) Monoclonal antibody against cell surface GRP78 as a novel agent in suppressing PI3K/AKT signaling, tumor growth, and metastasis. Clin Cancer Res 19:6802-6811

38. Rezanejhad L, Zolghadri J, Gharesi-Fard B (2013) Importance of antiGRP78 antibody in pre-eclampsia. Iran J Immunol 10:238-246

39. Zhang XX, Li HD, Zhao S, Zhao L, Song HJ, Wang G, Guo QJ, Luan ZD, Su RJ (2013) The cell surface GRP78 facilitates the invasion of hepatocellular carcinoma cells. Biomed Res Int 2013:917296

\section{Submit your next manuscript to BioMed Central and we will help you at every step:}

- We accept pre-submission inquiries

- Our selector tool helps you to find the most relevant journal

- We provide round the clock customer support

- Convenient online submission

- Thorough peer review

- Inclusion in PubMed and all major indexing services

- Maximum visibility for your research

Submit your manuscript at www.biomedcentral.com/submit
BioMed Central 